\title{
Equilibrium structure of dense gases
}

\author{
B. Sedunov \\ Computer and Information Systems Department, Russian New University, Moscow 105005, Russia
}

\section{Introduction}

The computer aided analysis of precise thermophysical data for pure real gases and denser fluids is developed here to provide a vision of their equilibrium molecular structure [1, 2]. The structure of fluids means the structural features of disordered systems. It differs from the regular structures of molecules or solids [3] and reflects the structural features of the temporarily existing local molecular complexes, such as clusters and pores of different dimensions.

Pure fluids are actively used in modern chemical technologies as initial and intermediate substances and final products. For further improvement of technological processes with pure fluids it is important to know better their molecular interaction mechanisms and parameters. The purity of gases and denser fluids by itself presents a great scientific value: it opens possibility to discover the details of the molecular interactions and the cluster structure by the computer aided processing of precise thermophysical data for pure substances.

The distribution of intermolecular distances in fluids is not uniform. The diminishing of the fluid density from its maximal value does not mean the uniform expansion of all intermolecular distances. Some of distances stay the same as in a dense liquid, but others become noticeably enlarged. In real gases some part of molecules forms the short distance complexes, named clusters. The clusters in real gases now attract a large attention of researches [48]. The Water vapor clusters are responsible for clouds formation in upper layers of atmosphere [9].

The factors complicating determination of the molecular interaction mechanisms [1]:

- Densely spaced and disarranged by thermal movement bound states in clusters;

- The plurality of the cluster isomer configurations, growing with a number of particles in a cluster;

- The difference between the thermodynamically averaged energetic level of the bound state and the minimal potential energy of a cluster;

- Long relaxation times for tightly bound cluster isomers preventing from a fast reaching the equilibrium and thus distorting the experimental data at low temperatures.

There are various approaches to molecular interaction mechanisms. The earlier models of fluids described in $[10,11]$, such as the famous van der Waals equation of state or the corresponding states law, have been based on the experimental data taken with not very high precision. They provided only a general picture of fluids sacrificing very important details of their molecular interactions. Now, the individual characteristics of molecular interactions in substances are being actively studied asing in the precise experimental data [12].

The constantly growing precision of thermophysical data opens new ways to penetrate deeper into details of the fluids' structure. But this task is not very easy. It may be referred to the class of reverse mathematical problems, the results of which widely diverge at slight changes of initial data. Therefore, we should use the methods of data processing capable to limit the level of this divergence by both the regularization of the initial data and selecting the appropriate set of the molecular interaction parameters. This set should be adjusted to real features of the molecular interactions in gases instead of the purely mathematical parameterization of experimental data.

An alternative approach to the molecular interactions in fluids is based on the simulations of the many particle systems behavior with different model potentials [4-9, 13]. The problem of this approach lies in rather arbitrary assumptions about the model potentials describing interactions between molecules in fluids. I.G. Kaplan [13] used more than 50 model potentials in his investigations. He came to very important conclusions for the dispersion forces temperature dependence.

The optimal way to the physically correct picture of fluids may be in the mutually correlated utilization of both approaches: from the experimental data and from the model potentials. These approaches should converge in the molecular interaction parameters to be found. That may serve as the criterion of the most appropriate model potential selection.

This work deals with the main thermodynamics principles extension to real gases and denser fluids and develops the methods of the molecular interaction parameters extraction from the experimental data. It is aimed at the cluster fractions' characteristics near the saturation $\mathrm{D}_{\text {sat }}$ and even critical $\mathrm{D}_{\mathrm{cr}}$ densities of pure gases basing on precise data from [15]. 


\section{The method of analysis}

To discover the cluster structure of gases a series expansion of thermophysical values by powers of the monomer fraction density [14], $\mathrm{D}_{\mathrm{m}}$, is recommended here, in accordance with the chemical thermodynamics principles.

Among different thermophysical values the most appropriate for the clusters' characterization is the potential energy U. In our phenomenological approach the molar potential energy $U$ of the fluid can be defined as the difference between the internal energies $E$ at the existing pressure $\mathrm{P}$ and zero pressure: $\mathrm{U}=\mathrm{E}(\mathrm{T}, \mathrm{P})-\mathrm{E}(\mathrm{T}, 0)$. Figure 1 shows a typical pressure dependence of the $U(P)$ in Propane at $\mathrm{T}=360 \mathrm{~K}$. The values for the internal energy have been taken from the NIST database [15].

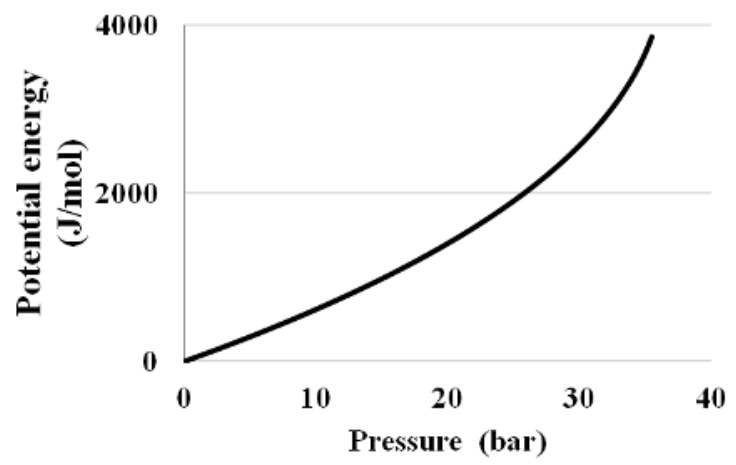

Figure 1. The pressure dependence of the potential energy in Propane at $\mathrm{T}=360 \mathrm{~K}$.

The potential energy is zero for the monomer fraction because the monomers do not interact with each other. Near zero $P$ the slope of the $U(P)$ curve is determined mainly by dimers, but at larger pressures the steepness of slope grows, thus reflecting the growing role of larger clusters. This quick growth complicates the analysis because it increases errors for estimated characteristics of larger clusters.

To estimate the clusters' bond parameters and their concentrations, the author recommends using the density UD of the potential energy $U$. The n-power term of this value series expansion by powers of $D_{m}$ at a constant $T$ provides the n-particle clusters' contribution in UD. The expansion coefficients are products of the clusters' equilibrium constants and bond energies.

\subsection{Thermophysical databases as a mirror of molecular interactions}

Now, the computer aided design and quality control for many technological processes is supported by comprehensive databases of thermophysical properties for substances to be used. In modern science and technology their creation involves the computer processing and generalization of multiple experimental data from all over the World with a steady growth of the data abundance and precision. The constant improvement of comprehensive and precise databases of the NIST [15, $16]$ is the best example of this kind of scientific activity.
The systematization of experimental data, from one side, provides practical application, testing and correction of the existing theoretical models and methods, but from the other side, opens possibilities for further development of the theoretical basis.

Unlike the printed handbooks, as [17], the modern online databases of equilibrium thermophysical properties accumulate and generalize data from different sources. They take into account the thermodynamically driven mutual correlations of different properties of fluids, such as the pressure-volume-temperature (PVT) and the energy-entropy-pressure-temperature

(EEPT) dependences. These correlations put into the interpolation polynomials effectively suppress occasional deviations of experimental data from regular dependences, thus providing possibilities for further computer aided processing of the data. It helps to expand the density range of gases under investigation for the extraction from the regularized data the bond parameters for larger clusters.

\subsection{The argument for series expansions of thermophysical values}

The developed computer aided processing of thermophysical data for pure fluids uses the monomer fraction density $D_{m}$ [14] as the main variable for series expansions of the equilibrium thermophysical values. $D_{m}$ is the number of moles per liter of those basic particles that are temporarily not bound and perform only elastic collisions with other particles of a gas.

The monomer fraction density is relative to the fugacity, accounting for the no ideality of gases, and can be found from the Equation (1) [14]:

$\partial \mathrm{D}_{\mathrm{m}} /\left.\partial \mathrm{P}\right|_{\mathrm{T}}=\mathrm{D}_{\mathrm{m}} /(\mathrm{RTD})$

The integration of this Equation requires small values both for the initial pressure and for the digital processing steps $[1,14]$. The Figure 2 compares the $D_{m}$ values in Fluorine at $\mathrm{T}=150 \mathrm{~K}$ with the total density $\mathrm{D}$ and the ideal gas density $\mathrm{D}_{\mathrm{p}}=\mathrm{P} / \mathrm{RT}$.

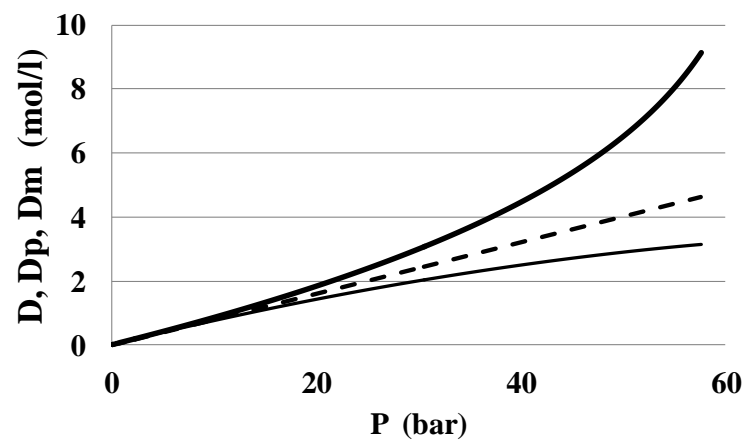

Figure 2. The monomer fraction density $\mathrm{D}_{\mathrm{m}}$ (thin line) as compared to the total density $\mathrm{D}$ (thick line) and the ideal gas density $\mathrm{D}_{\mathrm{p}}=\mathrm{P} / \mathrm{RT}$ (dashed line) for Fluorine at the temperature $\mathrm{T}=150 \mathrm{~K}$.

The Figure 2 shows that at high pressures the total density $\mathrm{D}$ greatly differs from the partial monomer fraction density, $D_{m}$. Therefore, the results of series 
expansions of thermophysical values by powers of D and $D_{m}$ are quite different. In terms of mathematics we should take $D_{m}$, but not $D$, for the boundary condition of the particles' population in the cluster's potential well!

The series expansion of thermophysical values by powers of the total density $\mathrm{D}$ is used in the virial expansion $[16,18]$ that provide the same results as the expansion by $\mathrm{D}_{\mathrm{m}}$ only for the second virial coefficient. The higher virial coefficients do not have a clear physical sense because they result from the expansions by powers of the total density $\mathrm{D}$ being the mixture of the partial densities. For this reason the higher virial coefficients are the combinations of smaller clusters' characteristics.

\subsection{The way to the molecular interaction parameters}

The potential energy density UD in pure real gases is a sum of partial values (UD) :

$\mathrm{UD}=\sum(\mathrm{UD})_{\mathrm{n}}=-\sum \mathrm{E}_{\mathrm{n}} \mathrm{D}_{\mathrm{n}}$.

Here $D_{n}$ are the molar densities of $n$-particle cluster fractions and $E_{n}$ - the effective bond energies of the $n$ particle clusters. The $E_{n}$ values may change with temperature due to the changing of the dominanting isomer configurations in cluster fractions $[1,2]$. The series expansion of UD starts from the second power, because the monomers do not contribute to the potential energy.

The bond energies $E_{n}$ of clusters in real gases are the averaged energies of the clusters' decomposition to monomers. To find the $E_{n}$ values we use the series expansion coefficients $K_{u n}(T)=-(U D)_{n} / D_{m}{ }^{n}$ and plot the logarithm of the $K_{u n}(T)$ versus the reverse temperature. In the zone of temperatures, where the graph is linear, the slope of the line gives the cluster bond energy in $\mathrm{K}$ (Figure 3).

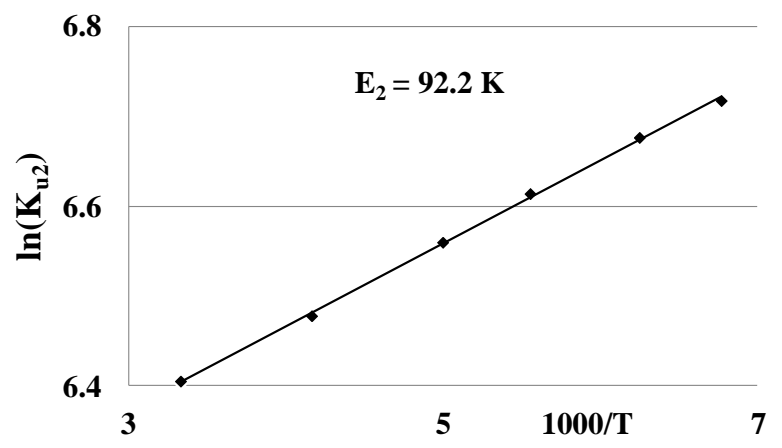

Figure 3. The pair bond energy, measured in $\mathrm{K},\left(\mathrm{E}_{2}=92.2\right.$ $\mathrm{K}$ ) at temperatures $148-300 \mathrm{~K}$ for dimers in the gaseous $\mathrm{CF}_{4}$.

\section{THE RESULTS}

\subsection{The temperature dependence of the pair bond energy}

The analysis of the pair bond energies for dimers in many gases discovers the temperature dependence of the $E_{2}(T)$, shown on the Figures 4-6.

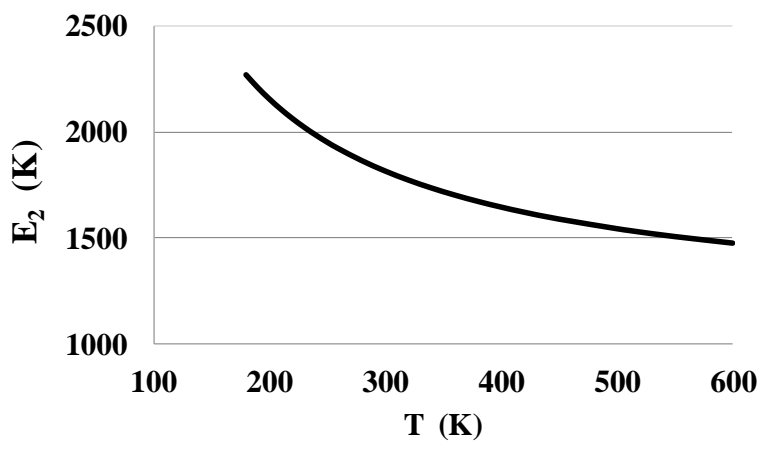

Figure 4. The pair bond energy for dimers in the Methanol vapor.

In polar gases, like Water or Methanol, it is quite natural, because the hydrogen bonds have a strong angular dependence of their interaction potential. The mutual angular movement of molecules in dimers may be freezed at lower temperatures, thus enlarging the $\mathrm{E}_{2}$ value. The transition from the low $\mathrm{T}$ to high $\mathrm{T}$ values for $\mathrm{E}_{2}$ in the Water vapor takes rather wide temperature range from 350 to $450 \mathrm{~K}$, Figure 5. Therefore, this transition is named as soft.

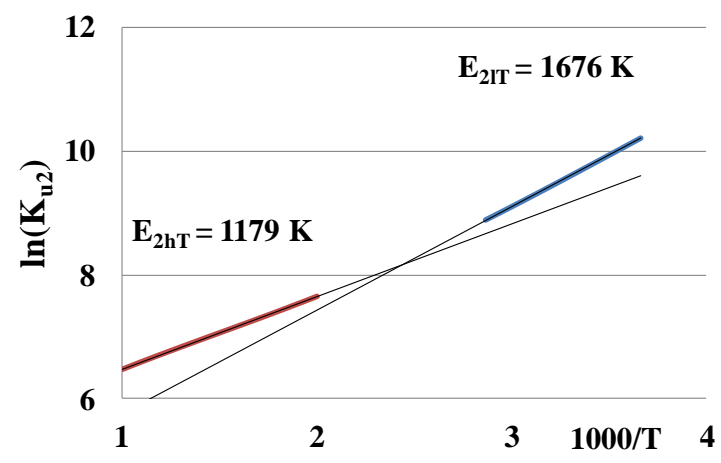

Figure 5. The change of the pair bond energy for dimers in the Water vapor.

But the dependence of the $\mathrm{E}_{2}(\mathrm{~T})$ in an atomic gas, like Krypton, Figure 6, seems to witness the existence of selected directions for the noble atoms bonding in dimers.

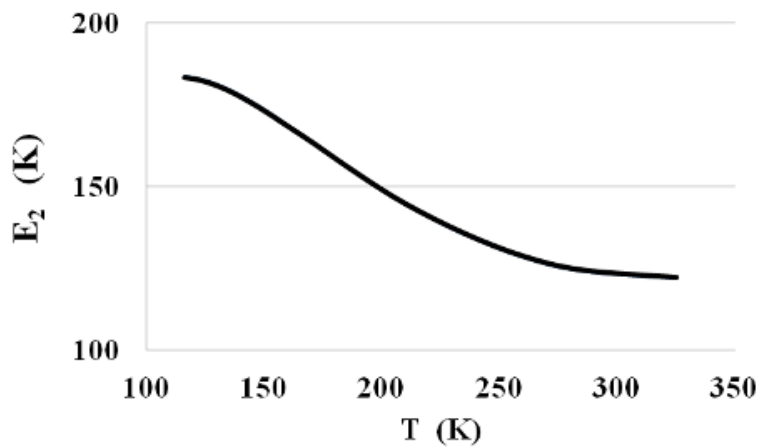

Figure 6. The pair bond energy for dimers in the Krypton gas.

\subsection{Soft structural transitions between dominating isomers of clusters}

The temperature dependences of the UD expansion coefficients $\mathrm{K}_{\mathrm{un}}$ for larger clusters in several gases exhibit 
the change of the cluster bond energy in some temperature range. This change may be seen clearly at the chart $\ln \left(K_{u n}\right)$ versus 1000/T, Figure 7.

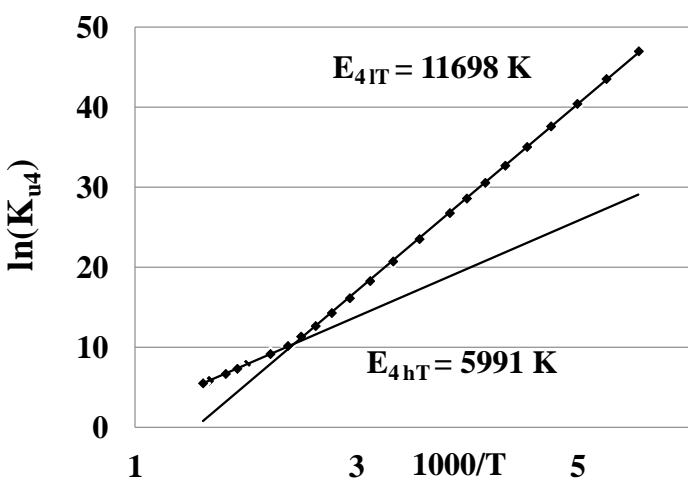

Figure 7. The soft structural transition of the Methanol vapor tetramers near $\mathrm{T}=420 \mathrm{~K}$.

The change of the $\mathrm{E}_{4}$ value for tetramers in the Methanol vapor shows on the structural transition in the tetramer fraction from tightly bonded isomers at low $\mathrm{T}$ to loosely bound isomers at $\mathrm{T}>420 \mathrm{~K}$. The temperature range of the soft structural transition for tetramers in Methanol, Figure 7, is much more narrow than for dimers in the Water vapor, Figure 5. It is obvious that this width falls with growing the $E_{n} / T$ ratio.

The structural transition in cluster fractions is not abrupt as it is seen from the Figure 8 that displays the soft melting of the Water tetramers in the temperature range 330-370 K.

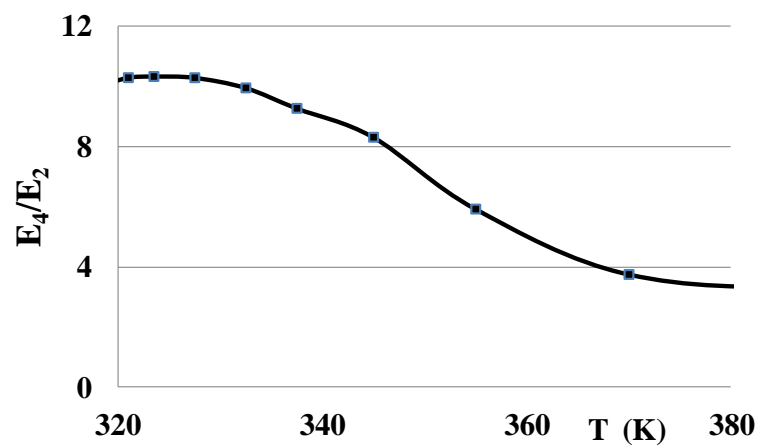

Figure 8. Soft structural transition in the Water vapor tetramers.

The soft melting of clusters is not a phase transition. It takes a range of temperatures because any cluster may change its structure independently on the other clusters.

But the first order phase transition from liquid to gas is a collective action of all molecules of the liquid phase with huge $E_{n} / T$ ratio, for this reason it is abrupt.

The tetramer to dimer bond energy ratio $E_{4} / E_{2}$ in the transition zone for Water vapor, Figure 9, changes from $8-10$ at $\mathrm{T}<330 \mathrm{~K}$ to $3.5-4$ for $\mathrm{T}>380 \mathrm{~K}$.

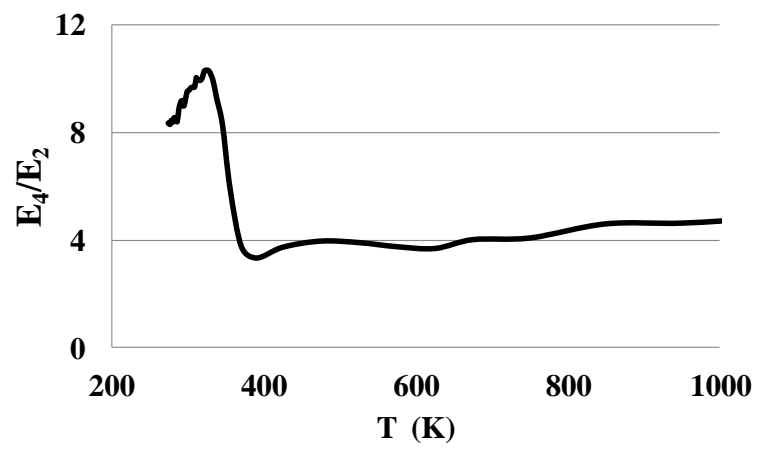

Figure 9. Soft melting of the Water vapor tetramers.

It tells that the dominating at hight temperatures 4particle isomers tend to the chain-like polymer or ring structure with 3-4 bonds between Water molecules. But at $\mathrm{T}<330 \mathrm{~K}$ the Water tetramers have a rigid tightly bonded structure equivalent to 8-10 pair bonds between molecules. In this case both Hydrogen atoms of every Water molecule may participate in the bonding of the tetramer. This interesting fact has to be studied by some alternative method, like spectroscopy.

\subsection{Loose and dense cluster fractions at high densities of gases}

The shown on the Figure 1 nonlinear growth of the potential energy $U$ at high pressures is much more increased after the multiplication $U$ by $D$. To start the series expansion of UD from the second coefficient $\mathrm{K}_{\mathrm{u} 2}$ we use the cluster bond function $\mathrm{W}=\mathrm{UD} / \mathrm{D}_{\mathrm{m}}{ }^{2}$.

Figure 10 shows the dependence on $D_{m}$ of the cluster bond function $\mathrm{W}=\mathrm{UD} / \mathrm{D}_{\mathrm{m}}{ }^{2}$ for Xenon gas at the supercritical temperature $\mathrm{T}=300 \mathrm{~K}\left(\mathrm{~T}_{\mathrm{cr}}=289.733 \mathrm{~K}\right.$ [15]).

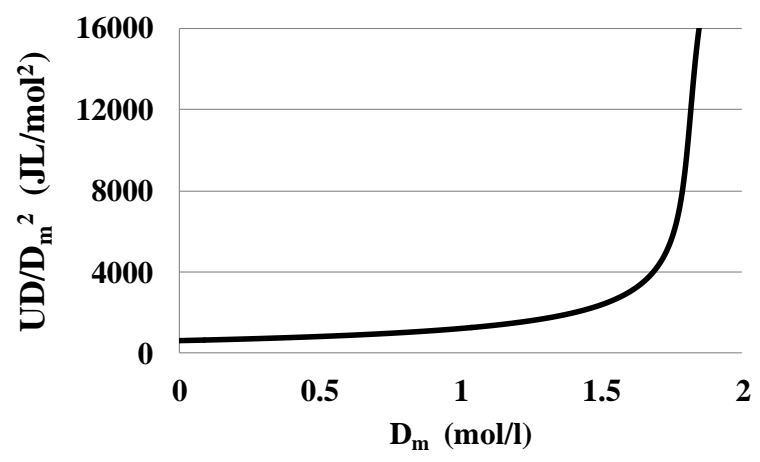

Figure 10. The cluster bond function $U D / \mathrm{D}_{\mathrm{m}}{ }^{2}$ for Xenon gas at the supercritical $\mathrm{T}=300 \mathrm{~K}$.

It is seen that near the critical density of the gas the cluster bond function $\mathrm{W}\left(\mathrm{D}_{\mathrm{m}}\right)$ goes up almost vertically. It makes the series expansion of this function impossible.

But for many pure gases the reverse function $\mathrm{D}_{\mathrm{m}}{ }^{2} /(\mathrm{UD})$ happens to depend on $\mathrm{D}_{\mathrm{m}}$ almost linearly in a wide range of densities [2] that simplifies the analysis and can be interpreted as the geometric progression for the expansion polynomial. In [2] it was demonstrated for Carbon Dioxide, here we illustrate this statement on the example of the Xenon high density gas, Figure 11. 


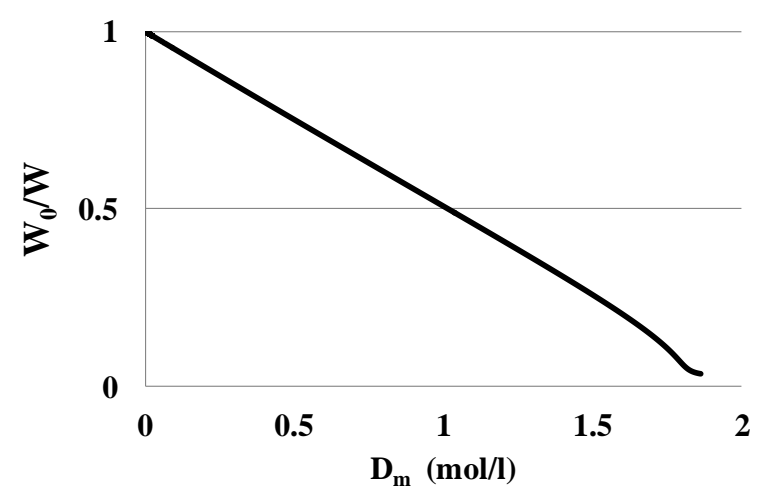

Figure 11. Almost linear reverse bond function $\mathrm{W}_{0} / \mathrm{W}$ for Xenon at the $\mathrm{T}=300 \mathrm{~K}$.

This progression corresponds to an infinite row of clusters of growing sizes with a universal mechanism of bonding. These clusters with relatively small bond energies can be named as loose clusters [2] and they possess the chain-like, polymer structure. The energy of a new particle bonding to the end of the loose cluster $\mathrm{E}_{\text {end }}$ can be found from the temperature dependence of the $\mathrm{W}_{0} / \mathrm{W}$ tangent of slope. This energy is slightly lower than the pair bond energy $E_{2}$, Figure 12.

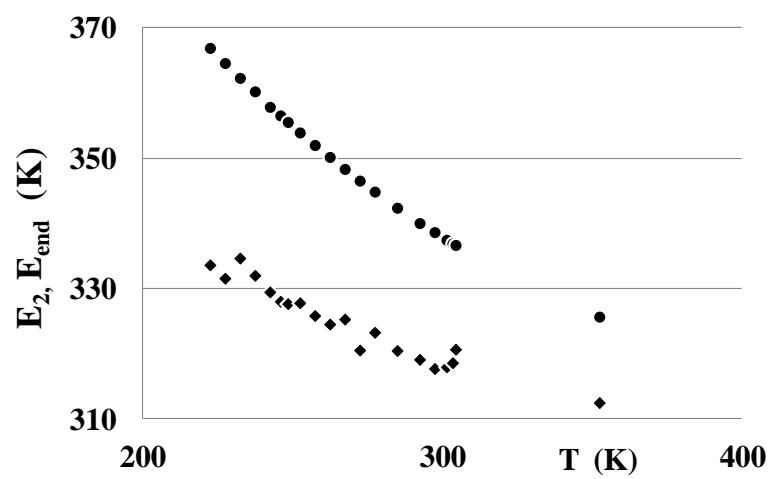

Figure 12. The pair bond energy $\mathrm{E}_{2}$ in the $\mathrm{CO}_{2}$ dimers (round markers) and the energy of a new particle bonding to the end of the linear chain cluster $\mathrm{E}_{\text {end }}$ (rhomb markers).

In the Water vapor this method does not work because of the tightly bonded tetramers distorting the linearity of the $\mathrm{W}_{0} / \mathrm{W}$ dependence on $\mathrm{D}_{\mathrm{m}}$.

The deviations from the straight line at higher densities, Figure 11, correspond to dense clusters with larger bond energies. For gases with a long linear part of the $\mathrm{W}_{0} / \mathrm{W}$ dependence on $\mathrm{D}_{\mathrm{m}}$ the row of dense clusters starts from the relatively large number of particles in the dense cluster and this row is not continuous, but jumping. For example, in Xenon the starting number for dense clusters is 7 and the next numbers at $\mathrm{T}=300 \mathrm{~K}$ are 10 , $14,26,60,133,150$.

\section{Conclusion}

- Basing on the precise online databases of thermophysical properties for pure fluids, like the NIST database [15], it is possible to discover the cluster structure of real gases and bond energies of the dominating isomers of clusters;
- The series expansion of the gaseous potential energy density by powers of the monomer fraction density permits to find the bond parameters of clusters;

- The found bond energies of clusters in some temperature ranges perform the step-wise changes of their values, thus reflecting the soft transitions from one dominating isomer to another;

- At high gas densities it is possible to find characteristics of the linear chain polymer-like loose clusters with a continuous row of the particle numbers and with a universal bonding mechanism for the end particles;

- The characterization of the loose clusters permits to estimate the properties of the tightly bonded dense clusters with a jumping row of the particle numbers.

\section{References}

1. B. Sedunov, J. of Thermodynamics Article ID 859047 (2012)

2. B. Sedunov, American J. of Analytical Chemistry 3 899 (2012)

3. IUPAC, Compendium of Chemical Terminology, Blackwell Scientific Publications, Oxford, UK, 2nd ed., The Gold Book". Compiled by A. D. McNaught and A. Wilkinson, 1997

4. I. Kusaka, D.W. Oxtoby J. Chem. Phys. 1105249 (1999)

5. I.J. Ford, J. Mechanical Engineering Science 218 (C) $883(2004)$

6. P. Schaaf, B. Senger, H.J. Reiss, Phys. Chem. B 101 8740 (1997)

7. D.I. Zhukhovitskii J. Chem. Phys. 1107770 (1999)

8. M.J. McGrath, J. Chem. Phys. 133084106 (2010)

9. A.Y. Zasetsky et al. Atmos. Chem. Phys. 9965 (2009)

10. J. Rowlinson Cohesion: A Scientific History of Intermolecular Forces. Cambridge University Press, 2002

11. R.C. Reid, J.M. Prausnitz, T.K. Sherwood, The properties of liquids and gases, McGraw Hill, New York, 1977

12. B.E. Poling, J.M. Prausnitz, J.P. O'Connell, The Properties of Gases and Liquids, $5^{\text {th }}$ ed., Mc GrawHill, NewYork, 2001

13. I.G. Kaplan Intermolecular Interactions: Physical Picture, Computational Methods and Model Potentials John Wiley \& Sons, Ltd, 2006

14. B. Sedunov, Int. J. of Thermodynamics 111 (2008)

15. NIST, Thermophysical Properties of Fluid Systems, 2013 http://webbook.nist.gov/chemistry/fluid

16. NIST Database of the Thermophysical Properties of Gases Used in the Semiconductor Industry, 2013 http://properties.nist.gov/fluidsci/semiprop/

17. N.B. Vargaftik, Yu. K. Vinogradov, V.S. Yargin, Handbook of physical properties of liquids and gases, Begell House Inc. 3-d ed., 1996

18. J.E. Mayer, G.M. Mayer, Statistical Mechanics, John Wiley and Sons, New York, 1977 